RASĀYAN J. Chem.

Vol. 13 | No. 3 |1357-1362| July - September | 2020 ISSN: 0974-1496 | e-ISSN: 0976-0083 | CODEN: RJCABP

\title{
PHOTOCATALYTIC APPLICATIONS OF SYNTHESISED AI GUM STABILIZED COBALT DOPED ZnO NANOPARTICLES
}

\author{
R. Sakthivel ${ }^{1}$, A. Geetha ${ }^{2}$, Ba. Anandh ${ }^{1}$ and A. Shankar Ganesh ${ }^{1}$ \\ ${ }^{1}$ Department of Electronics, PSG College of Arts \&Science, Coimbatore-14, India \\ ${ }^{2}$ Department of Chemistry, Hindusthan College of Arts \& Science, Coimbatore-14, India \\ E-mail: rsgvel@gmail.com
}

\begin{abstract}
In the present work, we have synthesized Azadirachta indica gum (AI gum) stabilized cobalt (Co) doping on $\mathrm{ZnO}$ (Co: $\mathrm{ZnO} \mathrm{NPs}$ ) using a conventional wet chemical method and structural, morphological, optical properties of obtained AI-Co: ZnO NPs were studied. Average particle size and shape of AI-Co: ZnO NPs were observed using high-resolution Schottky emitter FE-SEM. The surface morphological images of FE-SEM exhibit that all the particles were spherical. From UV-Visible Spectroscopy (UV) analysis, the absorption intensity was slightly shifted to lower wavelength due to variation in the cobalt concentration and the bandgap was increased with an increase in doping percentage of cobalt. The calculated band gap energy was $3.55 \mathrm{eV}, 3.57 \mathrm{eV}$ and $3.59 \mathrm{eV}$ for AI$\mathrm{Zn}_{0.97} \mathrm{Co}_{0.03} \mathrm{O}, \mathrm{AI}-\mathrm{Zn}_{0.95} \mathrm{Co}_{0.05} \mathrm{O}$ and $\mathrm{AI}-\mathrm{Zn}_{0.93} \mathrm{Co}_{0.07} \mathrm{O}$ nanoparticles, respectively. The chemical functional group or molecular structure of AI-Co: ZnO NPs was studied using Fourier Transform Infrared (FTIR) spectroscopy technique. The degradation of Methylene Blue (MB) was found to be $48 \%, 58 \%$ and $74 \%$ for $0.01 \mathrm{~g}, 0.02 \mathrm{~g}$ and 0.03 $\mathrm{g}$ of $0.5 \%$ cobalt doped AI gum stabilized Co-doped $\mathrm{ZnO}$ nanoparticles.
\end{abstract}

Keywords: ZnO, AI Gum, FESEM, FTIR, UV-Vis, Photocatalytic Activity.

(C) RASĀYAN. All rights reserved

\section{INTRODUCTION}

In recent years, nanoparticles materials created tremendous attention and played a vital role in various applications such as photocatalysis, optical field, electronics area, biotechnology, and environmental application. ${ }^{1}$ A green chemistry process can be defined as the development of a clean technology process which reduces the risk of the conventional chemical process. To reduce the organic pollutants in the ambiance, various metal oxides such as $\mathrm{TiO}_{2}, \mathrm{ZnO}, \mathrm{CuO}$, and $\mathrm{MgO}$ were used. ${ }^{2-3}$ Among these photocatalyst materials, various research articles are reported on the $\mathrm{ZnO}$ which act as an excellent photocatalyst due to their significant properties such as widest bandgap, non-toxic nature, low-cost material, and high electron mobility. ${ }^{4}$ The widest bandgap nature of $\mathrm{ZnO}$ applied in many fields and used for improving the efficiency of diodes, optoelectronics, chemical and biosensors and photocatalysts. ${ }^{5-8}$

Doping is an important method to turn the properties of semiconductors for specific applications. The physical and chemical properties of $\mathrm{ZnO}$ nanoparticles can be affected by doping in terms of the resulting structural, optical and photocatalytic properties. Many types of research revealed the impact of a transition metal such as zinc $(\mathrm{Zn})$, nickel $(\mathrm{Ni})$, cobalt $(\mathrm{Co})$, manganese $(\mathrm{Mn})$, etc., on the characterization of $\mathrm{ZnO}$ nanoparticles. Among these transition metals, various studies reported on the photoconductivity of cobalt (Co) doping on $\mathrm{ZnO}$ enhance the photocatalytic activity. ${ }^{9-12}$ Due to the excellent properties of cobalt, it considers as an advantageous material for doping $\mathrm{ZnO}$. Pascariu et al. addressed that cobalt doped $\mathrm{ZnO}$ samples show remarkable photocatalytic behavior. ${ }^{13}$ Rajbongshi and samdarshi reported that the hexagonal wurtzite phase of cobalt doped $\mathrm{ZnO}$ has the highest photocatalytic activity in visible light. ${ }^{14}$ Hence, we choose cobalt $(\mathrm{Co})$ doping into $\mathrm{ZnO}$ to increase the photocatalytic properties. In the present work, we have synthesized Azadirachta indica gum (AI gum) stabilized cobalt (Co) doping on $\mathrm{ZnO}$ (Co: $\mathrm{ZnO}$ NPs) using a simple wet chemical method and structural, morphological, optical properties of obtained Co: ZnO NPs were characterized. Finally, the photocatalytic application of the AI-Co: ZnO NPs were also studied.

Rasayan J. Chem., 13(3), 1357-1362(2020)

http://dx.doi.org/10.31788/RJC.2020.1335562

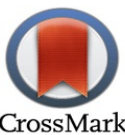


RASĀYAN J. Chem.

Vol. 13 | No. 3 |1357-1362 | July - September | 2020

\section{EXPERIMENTAL}

\section{Preparation of AI Gum stabilized Co: ZnO NPs}

In our work, Zinc nitrate hexahydrate $\left(\mathrm{Zn}\left(\mathrm{NO}_{3}\right)_{2} \cdot 6 \mathrm{H}_{2} \mathrm{O}\right)$ acts as a precursor material and cobalt(II)nitrate $\left(\mathrm{Co}\left(\mathrm{NO}_{3}\right)_{2} \cdot 6 \mathrm{H}_{2} \mathrm{O}\right)$ act as the doping material. Azadirachta indica gum (AI gum) is used as the stabilizing agent. All the experimental work was carried out using double distilled water as a solvent. To get a glassy mass of purified AI gum, Azadirachta indica gum exudates were washed with double distilled water to remove impurities. After complete dissolution of gum, it was filtered through Whatman filter paper (no: 40 ) and the filtrate was kept in a desiccator. High purity $\mathrm{Co}$ : $\mathrm{ZnO}$ nanoparticles have been prepared by conventional wet chemical treatment. $1 \mathrm{~g}$ of $\mathrm{Zn}\left(\mathrm{NO}_{3}\right)_{2} \cdot 6 \mathrm{H}_{2} \mathrm{O}$ was mixed in $90 \mathrm{ml}$ of highly purified double distilled water. The above-mixed solution was stirred using a magnetic stirrer for 10 minutes. $0.1 \mathrm{~g}$ of AI gum in $10 \mathrm{ml}$ water was added to the mixed solution. To this mixture, stoichiometric quantities of $\mathrm{Co}\left(\mathrm{NO}_{3}\right)_{2} \cdot 6 \mathrm{H}_{2} \mathrm{O}(0.03,0.05$, and 0.07$)$ were added using the formulae $\mathrm{Co}_{\mathrm{x}} \mathrm{Zn}_{(1-\mathrm{x})} \mathrm{O}$. This is continued by the addition of about $100 \mathrm{ml}$ of $\mathrm{NaOH}$ solution in dropwise with vigorous stirring for 3 hours. The final suspension with green precipitate obtained was centrifuged $10,000 \mathrm{rpm}$ for 3 minutes. The supernatant solution was discarded and the green precipitate obtained was dried at $100^{\circ} \mathrm{C}$ in hot air oven to remove the water molecules which are adsorbed on the surface of the synthesized nanoparticles. Finally, the green crystalline powder of AI-Co: ZnO NPs was obtained.

\section{Photocatalytic Activity}

Different concentrations $(0.01 \mathrm{~g}, 0.02 \mathrm{~g}$ and $0.03 \mathrm{~g}$ ) of AI gum stabilized 5\% Co-doped $\mathrm{ZnO}$ nanoparticles dispersed in $150 \mathrm{ppm}$ of methylene blue solutions were prepared. These suspensions were magnetically stirred in dark for 15 minutes and then irradiated using UV light. At different intervals of time, $2 \mathrm{ml}$ of suspension were extracted and centrifuged at the rate of 10,000 rpm for 3 minutes. Then the UV visible absorption spectrum was taken for the supernatant solution. Then the graph was plotted between degradation and concentration of dye, which is measured by the UV-VIS spectrometer.

\section{FE-SEM Analysis}

\section{RESULTS AND DISCUSSION}

The surface morphological properties and average particle size of AI-Co: ZnO NPs were analyzed using high-resolution Schottky emitter FE-SEM. Figure-1 exhibits the FE-SEM images of AI gum stabilized $\mathrm{Co}$ : ZnO NPs with different doping concentrations. It is observed that all the particles have a spherical shape. Increasing the doping concentration of $\mathrm{Co}$ into $\mathrm{ZnO}$ undergoes self agglomeration leading to an increase in particle size.

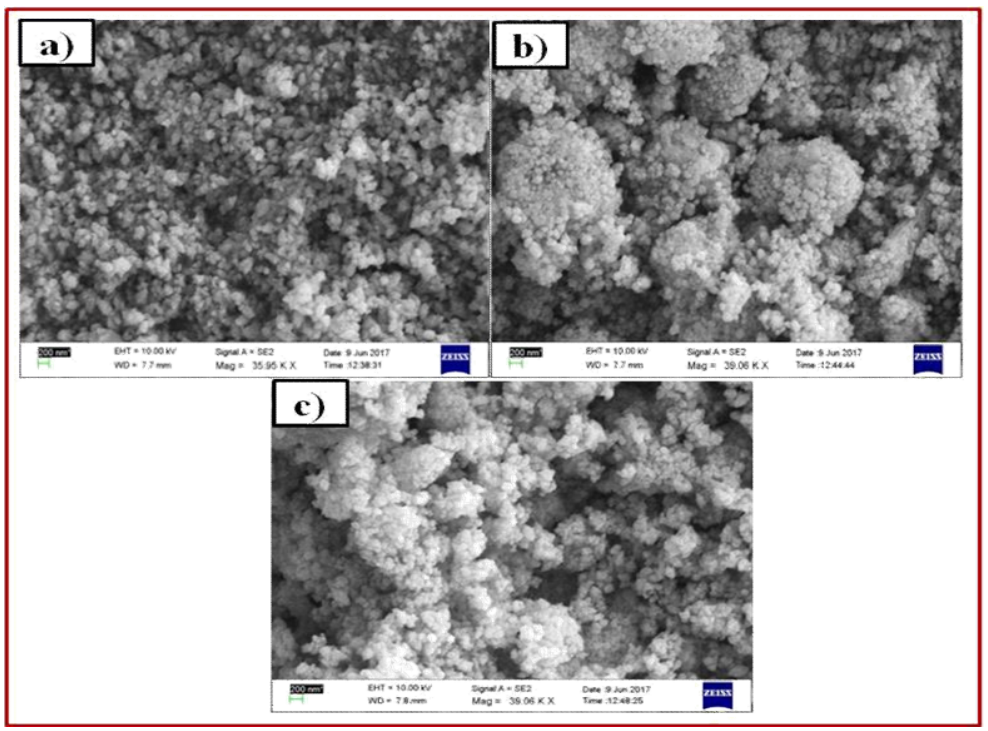

Fig.-1: FE-SEM Images of (a) $\mathrm{AI}-\mathrm{Zn}_{0.97} \mathrm{Co}_{0.03} \mathrm{O}$ (b) $\mathrm{AI}-\mathrm{Zn}_{0.95} \mathrm{Co}_{0.05} \mathrm{O}$ (c) $\mathrm{AI}-\mathrm{Zn}_{0.93} \mathrm{Co}_{0.07} \mathrm{O}$ Nanoparticles 
RASĀYAN J. Chem.

Vol. 13 | No. 3 |1357-1362| July - September | 2020

\section{UV-Visible Absorption Spectra Analysis}

The influence of cobalt doping on the optical properties of ZnO NPs is well defined in the UV-Vis absorption spectrum analysis. As observed in Fig.-2, the absorption intensity was slightly shifted to lower wavelength due to variation in the cobalt concentration.

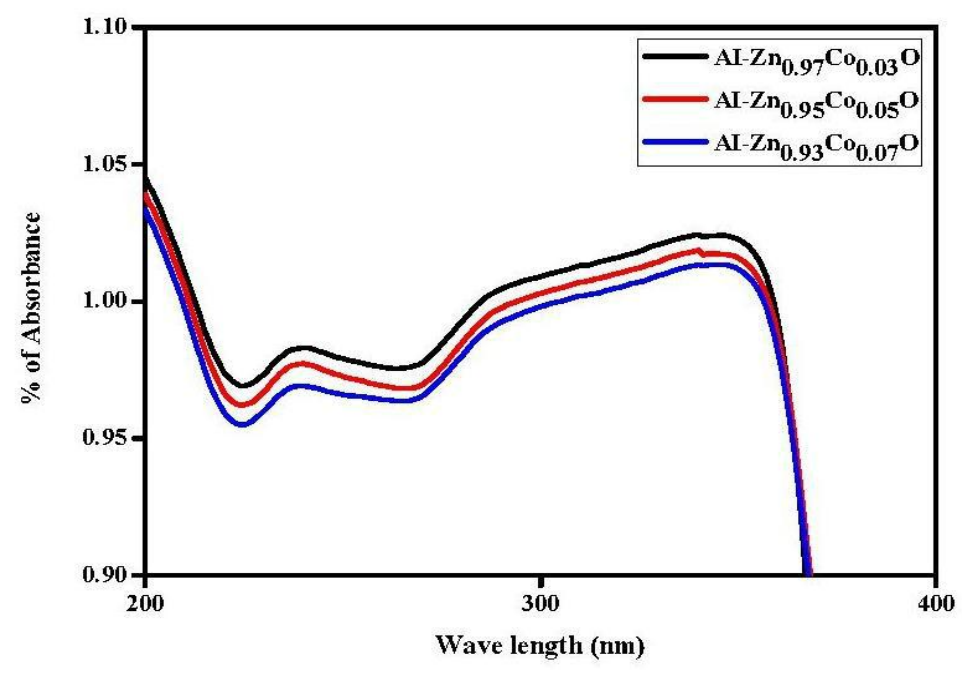

Fig.-2: Absorption Analysis of AI Gum stabilized Co: ZnO NPs

The absorption wavelength and calculated bandgap energy of the $\mathrm{AI}-\mathrm{Zn}_{0.97} \mathrm{Co}_{0.03} \mathrm{O}, \mathrm{AI}-\mathrm{Zn}_{0.95} \mathrm{Co}_{0.05} \mathrm{O}$ and $\mathrm{AI}-\mathrm{Zn}_{0.93} \mathrm{Co}_{0.07} \mathrm{O}$ nanoparticles are included in Table-1.

Table-1: Absorption Wavelengths and Bandgap Energy of AI Gum stabilized Co: ZnO NPs

\begin{tabular}{c|c|c|c}
\hline S. No. & Composition & Wavelength(nm) & Bandgap (eV) \\
\hline 1. & $\mathrm{AI}-\mathrm{Zn}_{0.97} \mathrm{Co}_{0.03} \mathrm{O}$ & 349 & 3.55 \\
\hline 2. & $\mathrm{AI}-\mathrm{Zn}_{0.95} \mathrm{Co}_{0.05} \mathrm{O}$ & 347 & 3.57 \\
\hline 3. & $\mathrm{AI}-\mathrm{Zn}_{0.93} \mathrm{Co}_{0.07} \mathrm{O}$ & 345 & 3.59 \\
\hline
\end{tabular}

As observed in Table-1 when increasing the Cobalt doping percentage, the value of the bandgap gets increased. On the other hand, the wavelength of the absorption gets decreased. A small shift in the absorption spectra occurs due to an increase in crystal size from 16 to $18 \mathrm{~nm}$. Previous reports also observed based on the changes in the bandgap value as the Cobalt doping percentage is increased. The pulsed laser deposited $\mathrm{Co}$ : $\mathrm{ZnO}$ thin films with $0 \%$ to $30 \%$ of doping showed that the bandgap value increased from $3.28 \mathrm{eV}$ to $3.80 \mathrm{eV}$ reported by Ivill. ${ }^{15}$ On the other hand, kim et al. addressed that the increasing Co doping percentage from 0 to $10 \%$, the value of the bandgap was decreased. ${ }^{16}$ The calculated band gap energy was $3.55 \mathrm{eV}, 3.57 \mathrm{eV}$ and $3.59 \mathrm{eV}$ for $\mathrm{AI}-\mathrm{Zn}_{0.97} \mathrm{Co}_{0.03} \mathrm{O}, \mathrm{AI}-\mathrm{Zn}_{0.95} \mathrm{Co}_{0.05} \mathrm{O}$ and $\mathrm{AI}-\mathrm{Zn}_{0.93}$ $\mathrm{Co}_{0.07} \mathrm{O}$ nanoparticles respectively.

\section{FTIR Spectroscopy Analysis}

To confirm the purity and behavior of the nanoparticles Fourier Transform Infrared (FTIR) spectroscopy technique was used to analyze the functional groups present in the synthesized nanoparticles. The interactions of AI gum with the synthesized nanoparticles are confirmed by this method. Figure- 3 shows the FTIR spectra of AI gum stabilized Co: ZnO NPs at room temperature.

The stretching mode of O-H group exhibits the existence of a little amount of water absorbed by the $\mathrm{ZnO}$ nanostructures hence the broad absorption band arises at around $3400-3500 \mathrm{~cm}^{-1}$ and also it reveals that the hydroxyl groups in the of AI gum present as a thin layer around the nanoparticles and avoids the agglomeration. ${ }^{17}$ The peaks at 1620,1632 and $1647 \mathrm{~cm}^{-1}$ are ascribed to the $>\mathrm{C}=\mathrm{O}$ bond. The band appeared in the range of $400-500 \mathrm{~cm}^{-1}$ is associated with metal oxide bond $(\mathrm{ZnO}) .{ }^{18-19}$ The frequency of 
RASĀYAN J. Chem.

Vol. 13 | No. 3 |1357-1362 | July - September | 2020

the $\mathrm{Zn}-\mathrm{O}$ bond is shifted towards higher wavenumber side in the spectrum of $\mathrm{AI}-\mathrm{Zn}_{0.97} \mathrm{Co}_{0.03} \mathrm{O}$, AI$\mathrm{Zn}_{0.95} \mathrm{Co}_{0.05} \mathrm{O}$ and $\mathrm{AI}-\mathrm{Zn}_{0.93} \mathrm{Co}_{0.07} \mathrm{O}$. This shift may be due to the replacement of $\mathrm{Zn}$ (atomic mass $=65.3$ ) with $\mathrm{Co}$ (atomic mass $=58.9$ ) by doping. There is no considerable shift in the $\mathrm{C}=\mathrm{N}$ and a higher shift of wavenumber in the $\mathrm{C}-\mathrm{O}$ band. The reduced mass of Co which is less than $\mathrm{Zn}$ may be the reason for shifting the peak towards the higher wavenumber side. There are no peaks related to cobalt oxide that were observed in the spectra, which suggests that Co ions were substituted in the regular lattice site of $\mathrm{ZnO}$. The absorption frequencies present in the samples are also shown in Table-2.

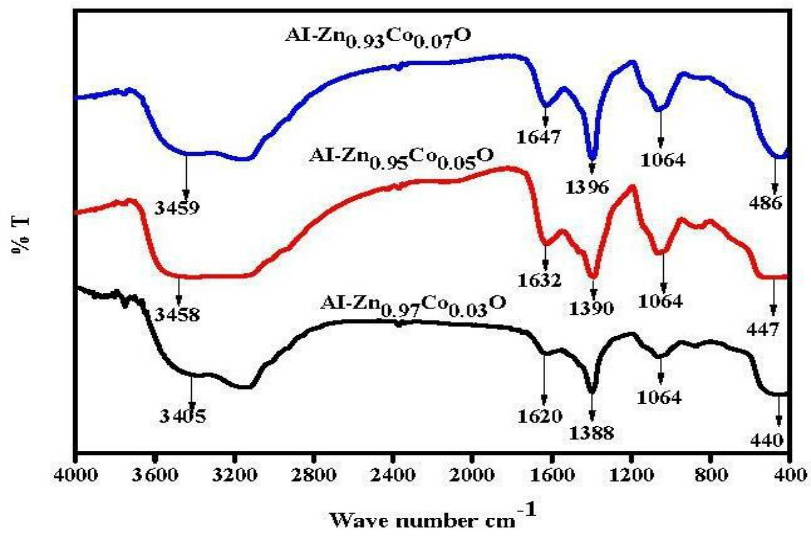

Fig.-3: FTIR Spectra of AI Gum stabilized Co: ZnO NPs

Table-2: Absorption Frequency of AI Gum stabilized Co: ZnO NPs

\begin{tabular}{c|l|c|c|c}
\hline \multirow{2}{*}{ S. No. } & \multirow{2}{*}{ Functional Groups } & \multicolumn{2}{|c}{ Absorption Frequency of Co Doped $\mathrm{ZnO}$ Nanoparticles(cm } \\
\cline { 3 - 5 } & & $\mathrm{AI}-\mathrm{Zn}_{0.97} \mathrm{Co}_{0.03} \mathrm{O}$ & $\mathrm{AI}-\mathrm{Zn}_{0.95} \mathrm{Co}_{0.05} \mathrm{O}$ & $\mathrm{AI}^{-} \mathrm{Zn}_{0.93} \mathrm{Co}_{0.07} \mathrm{O}$ \\
\hline 1 & $\mathrm{O}-\mathrm{H}$ (hydroxyl ) & 3405 & 3458 & 3459 \\
\hline 2 & $>\mathrm{C}=\mathrm{O}$ (carbonyl) & 1620 & 1632 & 1647 \\
\hline 3 & $\mathrm{~N}-\mathrm{H}$ (amine) & - & - & - \\
\hline 4 & $\mathrm{C}-\mathrm{N}$ (amide) & 1388 & 1390 & 1396 \\
\hline 5 & $\mathrm{C}-\mathrm{O}$ & 1064 & 1064 & 1064 \\
\hline 6 & $\mathrm{Zn}-\mathrm{O}$ & 440 & 447 & 486 \\
\hline
\end{tabular}

\section{Photocatalytic Applications}

The photocatalytic reaction was carried out using different concentrations of AI-Co: ZnO NPs in $100 \mathrm{ml}$ of $150 \mathrm{ppm}$ methylene blue solution. The Fig.-4(a-c) displayed the variation in the peak intensity of the absorption concerning wavelength as a function of time. As seen in the figures, it is observed that the peak intensity of the absorption was decreased when the time is increased for all the AI-Co: ZnO NPs. The absorption intensity was almost disappeared at $150 \mathrm{~min}$ irradiation time of UV light, hence this proves the nanoparticles used are highly photoactive. Figure- 5 shows the degradation efficiency of 0.01 , 0.02 , and $0.03 \mathrm{~g}$ of $\mathrm{AI}-\mathrm{Zn}_{0.95} \mathrm{Co}_{0.05} \mathrm{O}$ nanoparticles. It is observed that when time gets increased, the degradation efficiency is also increased linearly in all the AI-Co: ZnO NPs.

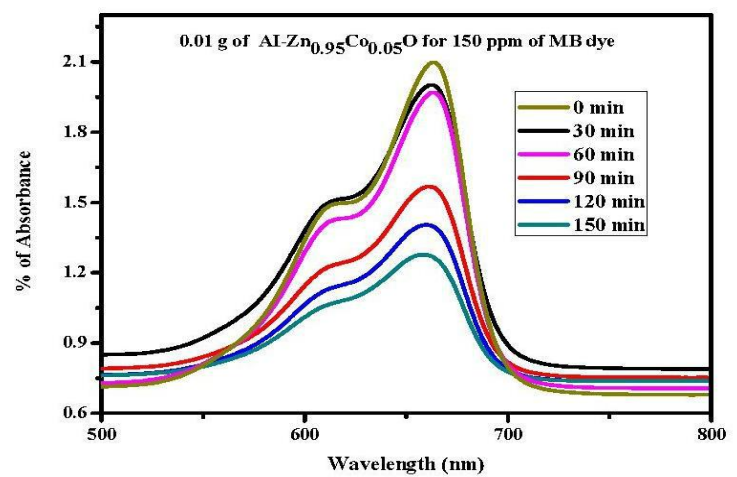

(a)

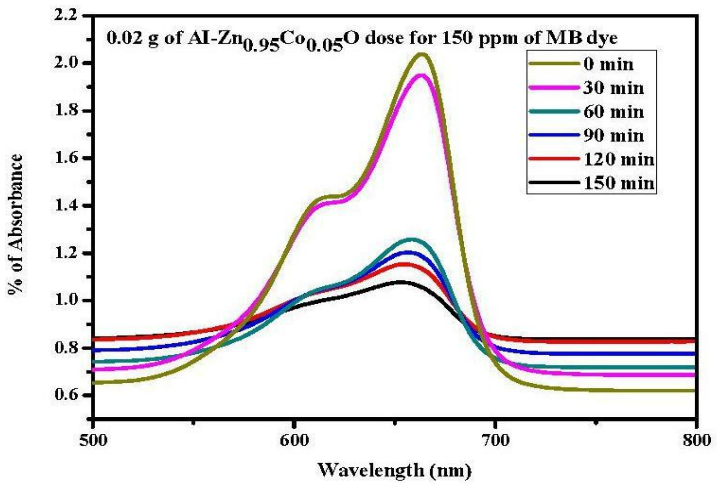

(b) 
RASĀYAN J. Chem.

Vol. 13 | No. 3 |1357-1362| July - September | 2020

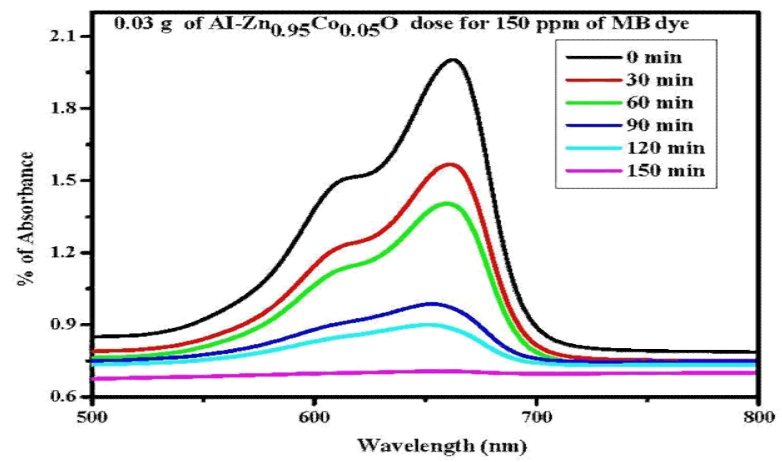

(c)

Fig.-4: Absorption Spectra of Methylene Blue for (a) $0.01 \mathrm{~g}$ of AI- $\mathrm{Zn}_{0.95} \mathrm{Co}_{0.05} \mathrm{O}$ Nanoparticles (b) $0.02 \mathrm{~g}$ of AI$\mathrm{Zn}_{0.95} \mathrm{Co}_{0.05} \mathrm{O}$ Nanoparticles (c) $0.03 \mathrm{~g}$ of $\mathrm{AI}-\mathrm{Zn}_{0.95} \mathrm{Co}_{0.05} \mathrm{O}$ Nanoparticles

The $0.03 \mathrm{~g}$ of AI gum stabilized Co-doped ZnO sample showed the highest photocatalytic behavior when compared to other samples and is following earlier reports. ${ }^{20-22}$ increasing the catalyst load from 0.01 to $0.03 \mathrm{~g}$ increases the surfaces to volume ratio of the photocatalyst and due to the availability of more active sites react with dye molecules which increases the photocatalytic activity

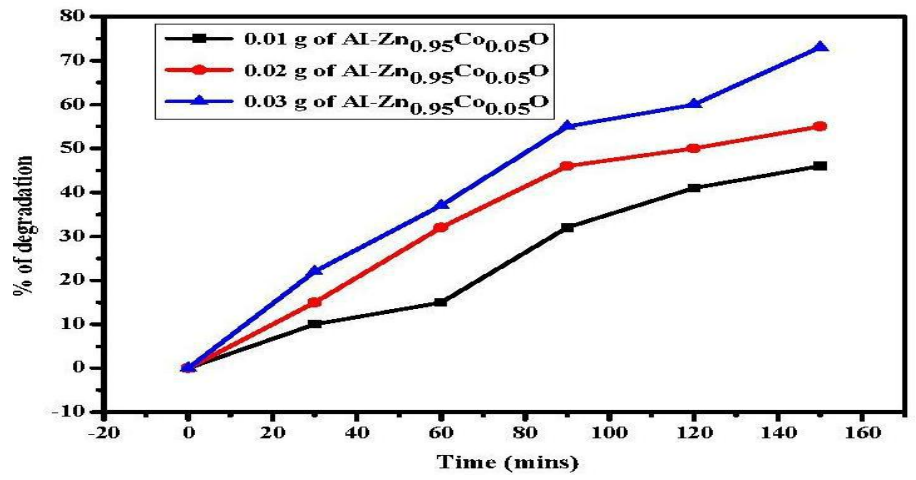

Fig.-5: Degradation Efficiencies of 0.01, 0.02 and $0.03 \mathrm{~g}$ of $\mathrm{AI}-\mathrm{Zn}_{0.95} \mathrm{Co}_{0.05} \mathrm{O}$ Nanoparticles for Methylene Blue Dye

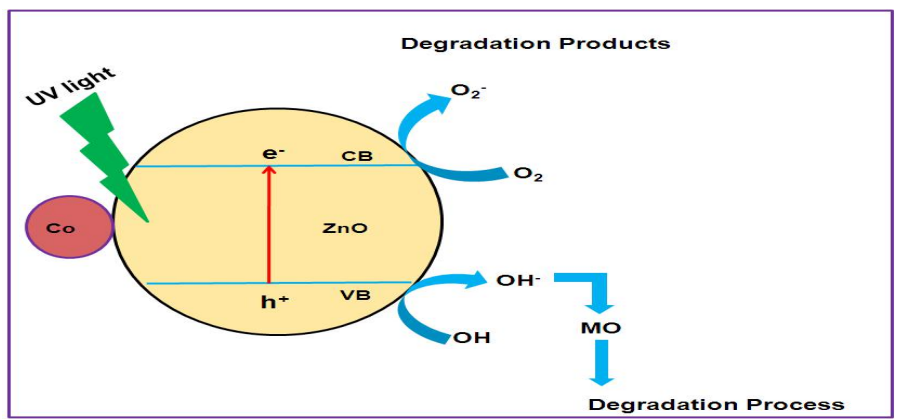

Fig.-6: Photocatalytic Mechanism of AI Gum stabilized Co-doped ZnO Nanoparticles Under UV Irradiation

In the case of $\mathrm{AI}$ gum stabilized $\mathrm{Co}: \mathrm{ZnO}(0.03 \mathrm{~g})$, the highest concentration may be a reason to increase in absorption of Methylene dye. After 150 minutes of testing, the degradation of $\mathrm{MB}$ was found to be $48 \%, 58 \%$ and $74 \%$ for $0.01,0.02$ and $0.03 \mathrm{~g}$ of $\mathrm{AI}-\mathrm{Zn}_{0.95} \mathrm{Co}_{0.05} \mathrm{O}$ nanoparticles respectively. The possible photocatalyst mechanism has given in Fig.6. The general mechanism of photocatalytic activity of AI gum stabilized cobalt doped $\mathrm{ZnO}$ nanoparticles, the electrons in the valence band (VB) of zinc oxide is first excited to its conduction band and the excited electrons are trapped by doped $\mathrm{Co}^{2+}$. The remaining hole in $\mathrm{ZnO}$ combines with water or hydroxyl ion to produce the hydroxyl radical, which causes degradation of MB dye molecules. The electrons that are originated from the VB of zinc oxide would thus be transported to the adsorbed oxygen from the air, and the recombination of electron and holes are avoided, which are 
RASĀYAN J. Chem.

Vol. 13 | No. 3 |1357-1362| July - September | 2020

all the beneficial reason for enhancing the UV-visible light-induced photocatalytic activity of the Codoped $\mathrm{ZnO}$ catalysts.

\section{CONCLUSION}

In our work, AI gum stabilized Co: ZnO NPs with different doping concentrations were successfully synthesized using a conventional wet chemical process. The Azadirachta indica gum acts as a good stabilizing agent and narrows the size of the nanoparticles. The crystalline structure and surface morphology were also not affected by the Co doping. FE-SEM results confirmed that the average particle size was increased with increasing the Co doping with a spherical shape. The incorporation of Co into AI gum stabilized $\mathrm{ZnO}$ was further confirmed by $\mathrm{UV}$ analysis. The calculated band gap energy was $3.55 \mathrm{eV}$, $3.57 \mathrm{eV}$, and $3.59 \mathrm{eV}$ for $\mathrm{AI}-\mathrm{Zn}_{0.97} \mathrm{Co}_{0.03} \mathrm{O}, \mathrm{AI}-\mathrm{Zn}_{0.95} \mathrm{Co}_{0.05} \mathrm{O}$ and $\mathrm{AI}-\mathrm{Zn}_{0.93} \mathrm{Co}_{0.07} \mathrm{O}$ nanoparticles respectively. The presence of a chemical functional group and the interaction of nanoparticles with AI gum were identified using FTIR analysis. The enhanced photocatalytic activity of AI gum stabilized Co: $\mathrm{ZnO}$ NPs towards MB dye solution under UV light was attributed to their high surface area leading to enhanced dye adsorption.

\section{REFERENCES}

1. Y. Liu, J. Han, W. Qiu, W. Gao, Applied Surface Science 263, 389(2012), DOI: 10.1016/j.apsusc.2012.09.067

2. Y.R. Zhang, J. Wan, Y.U. Ke, Journal of Hazardous Materials, 177, 750(2010), DOI: 10.1016/j.jhazmat.2009.12.095

3. Z. Han, L. Liao, Y. Wu, H. Pan, S. Shen, J. Chen, Journal of Hazardous Materials, 217-218, 100(2012), DOI:10.1016/j.jhazmat.2012.02.074

4. V. Etacheri, R. Roshan, V. Kumar, ACS Applied Materials and Interfaces, 4, 2717(2012), DOI: 10.1021/am300359h

5. F. Yakuphanoglu, Microelectronics Reliability, 51, 2195(2011), DOI:10.1016/j.microrel.2011.05.013

6. C.F. Wang, B. Hu, H.H. Yi, Optik, 123, 1040(2012), DOI:10.1016/j.ijleo.2011.07.027

7. S.K.Arya, S.Saha,J.E.R. Vick,V.Gupta, S. Bhansali, S.P. Singh, Analytica Chimica Acta, 737, 1(2012), DOI: 10.1016/j.aca.2012.05.048

8. H. Sutanto, I. Nurhasanah, E. Hidayanto, Materials Science Forum, 827,3(2015), DOI: $10.4028 /$ www.scientific.net $/ \mathrm{msf} .827 .3$

9. M. Yan, H.T. Zhang, E.J. Widjaja, R.P.H. Chang, Journal of Applied Physics, 94, 5240(2003), DOI: $10.1063 / 1.1608473$

10. Huihu Wang, Seonghoon Baek, Jaejin Song, Jonghyuck Lee, Sangwoo Lim, Nanotechnology, 19, 075607(2008), DOI: 10.1088/0957-4484/19/7/075607

11. P. Koidl, Physical Review B, 15, 2493(1977), DOI:10.1103/PhysRevB.15.2493

12. A. Johnson, A. Cohn, T. Kaspar, S.A. Chambers, G.M. Salley, D.R. Gameli, Physical Review B, 84, 125203(2011), DOI:10.1103/PhysRevB.84.125203

13. P. Pascariu, I.V. Tudose, M. Suchea, E. Koudoumas, N. Fifere, A. Airinei, Applied Surface Science, 448, 481(2018), DOI:10.1016/j.apsusc.2018.04.124

14. B. M. Rajbongshi, S.K. Samdarshi, Applied Catalysis B, 144, 435(2014), DOI:10.1016/j.apcatb.2013.07.048

15. M Ivill et al., New Journal of Physics, 10, 065002 (2008), DOI:10.1088/1367-2630/10/6/065002

16. K. J. Kim and Y. R. Park, Applied Physics Letters, 81(8), 1420(2002), DOI:10.1063/1.1501765

17. Y.J. Kwon, K. H. Kim, C.S. Lim. Journal of Ceramic Processing Research, 3, 146(2002).

18. R. F. Silva, M.E.D. Zaniquelli, Colloids and Surfaces A: Physicochemical and Engineering Aspects, 198-200, 551(2002), DOI:10.1016/S0927-7757(01)00959-1

19. Rasika Tankhiwale, S.K. Baipai, Colloids and surfaces B: Biointerfaces, 90, 16(2012), DOI:10.1016/j.colsurfb.2011.09.031

20. Y. Lu, Y. Lin, D. Wang, L. Wang, T. Xie, T. Jiang, Nano Research, 4, 1144(2011), DOI: $10.1007 / \mathrm{s} 12274-011-0163-4$

21. Q. Xiao, J. Zhang, C. Xiao, X. Tan, Material Science Engineering B, 142, 121(2007), DOI:10.1016/j.mseb.2007.06.021

[RJC-5562/2019] 